2. P. Davis, \& P. Rabinowitz, "Additional abscissas and weights for Gaussian quadrature of high order: values for $n=64,80$, and 96," J. Res. Nat. Bur. Standards, v. 60, 1958, p. $613-614$.

3. H. J. GAwLIK, "Zeros of Legendre polynomials of orders 2-64 and weight coefficients of Gauss quadrature formulae," Armament Research and Development Establishment Memoran$\operatorname{dum}(B) 7 \gamma / 58$, Fort Halstead, Kent, 25 p., Dec. 1958.

4. L. V. Kantorovich, \& V. I. KRYLOv, Approximate Methods of Higher Analysis, Interscience \& Noordhoff, New York and Groningen, 1958.

\title{
Bi-Variate, Rectangular, Optimum-Interval Interpolation
}

\section{By Ferdinand Freudenstein}

1. Definition of "Optimum-Interval" Interpolation. Let the real function $f(x, y)$, defined in the interval $-h \leqq x \leqq h,-k \leqq y \leqq k, h>0, k>0$, and assumed to have continuous partial derivatives of order up to $(m+n+2)$ in the rectangle, be approximated by the polynomial

$$
P_{m n}(x, y)=\sum_{r=0}^{m} \sum_{8=0}^{n} a_{r s} x^{r} y^{8}
$$

so that at the $(m+1)(n+1)$ precision points, $\left(x_{u}, y_{v}\right), u=0,1,2, \cdots m$; $v=0,1,2, \cdots n$,

$$
P_{m n}\left(x_{u}, y_{v}\right)=f\left(x_{u}, y_{v}\right)
$$

In that case,

$$
f(x, y)=P_{m n}(x, y)+R_{m n}
$$

where the remainder, $R_{m n}$, is given by the expression (see [1])

$$
\begin{array}{r}
R_{m n}=\frac{1}{(m+1) !} \prod_{u=0}^{m}\left(x-x_{u}\right) \frac{\partial^{m+1} f(\xi, y)}{\partial \xi^{m+1}}+\frac{1}{(n+1) !} \prod_{v=0}^{n}\left(y-y_{v}\right) \frac{\partial^{n+1} f(x, \eta)}{\partial \eta^{n+1}} \\
-\frac{1}{(m+1) !(n+1) !} \prod_{u=0}^{m}\left(x-x_{u}\right) \prod_{v=0}^{n}\left(y-y_{v}\right) \frac{\partial^{(m+1)+(n+1)} f\left(\xi^{\prime} \eta^{\prime}\right)}{\partial \xi^{\prime m+1} \partial \eta^{\prime n+1}}
\end{array}
$$

where $\xi, \xi^{\prime}\left(\eta, \eta^{\prime}\right)$ lie between the greatest and least of the numbers $x, x_{u}\left(y, y_{v}\right)$.

Consider the set, $S$, of all precision points $\left(x_{u}, y_{v}\right)$ within the rectangle $(-h, h)$ $(-k, k)$-called $(h, k)$ from now on. $S$ will be called the spacing. Let $h=k \delta$, where $\delta$ is a positive number defining the proportions of the rectangle. Furthermore, we restrict ourselves to functions for which the partial derivatives $\frac{\partial^{m+1} f(x, y)}{\partial x^{m+1}}, \frac{\partial^{n+1} f(x, y)}{\partial y^{n+1}}$, $\frac{\partial^{(m+1)+(n+1)} f(x, y)}{\partial x^{m+1} \partial y^{n+1}}$ are not equal to zero within $(h, k)$. Let $R$ be the largest lower limit on the maximum absolute value of $R_{m n}$ regardless of spacing. It is assumed

Received September 5, 1958; final revision April 22, 1960. 
that $f(x, y)$ is not of the form $P_{m n}(x, y)$; thus $R>0$. A particular spacing, $S_{0}$, will be determined with the property

$$
\lim _{h \rightarrow 0}\left(\frac{\text { Maximum absolute value of } R_{m n} \text { with spacing } S_{0}}{R}\right)=1 \text {, }
$$

$\delta$ and all $\frac{x_{u}}{h}, \frac{y_{v}}{k}$ remaining fixed. The spacing $S_{0}$ is called an optimum spacing. It should be observed that for finite values of $h$, the maximum absolute value of the remainder will in general not be minimized exactly, because of the unpredictable nature of the variation of the partial derivatives in equation (4).

2. Optimum-Interval Determination. Let

$$
\begin{aligned}
c_{\xi}=\frac{1}{(m+1) !} \frac{\partial^{m+1} f(\xi, y)}{\partial \xi^{m+1}} ; \quad c_{\eta} & =\frac{1}{(n+1) !} \frac{\partial^{n+1} f(x, \eta)}{\partial \eta^{n+1}} ; \\
& \text { and } \quad c_{\xi^{\prime} \eta^{\prime}}=\frac{1}{(m+1) !(n+1) !} \frac{\partial^{(m+1)+(n+1)} f\left(\xi^{\prime} \eta^{\prime}\right)}{\partial \xi^{\prime m+1} \partial \eta^{\prime n+1}} .
\end{aligned}
$$

Then

$$
\frac{c_{\xi^{\prime} \eta^{\prime}}}{c_{\xi} c_{\eta}} R_{m n}=P+Q+P Q
$$

where

$$
\begin{aligned}
& P=P\left(x, \xi^{\prime}, \eta^{\prime}, \eta\right)=\frac{c_{\xi^{\prime} \eta^{\prime}}}{c_{\eta}} \prod_{u=0}^{m}\left(x-x_{u}\right) ; \\
& Q=Q\left(y, \xi^{\prime}, \eta^{\prime}, \xi\right)=\frac{c_{\xi^{\prime} \eta^{\prime}}}{c_{\xi}} \prod_{v=0}^{n}\left(y-y_{v}\right) .
\end{aligned}
$$

As was assumed above, the partial derivatives are not equal to zero, so that their bounds can be identified as follows:

$$
a_{1} \leqq\left|c_{\xi}\right| \leqq a_{2} ; b_{1} \leqq\left|c_{\eta}\right| \leqq b_{2} ; c_{1} \leqq\left|c_{\xi^{\prime} \eta^{\prime}}\right| \leqq c_{2}
$$

Since

$$
\prod_{u=0}^{m}\left(x-x_{u}\right) \leqq(2 h)^{m+1} \text { and } \prod_{v=0}^{n}\left(y-y_{v}\right) \leqq(2 k)^{n+1}
$$

the maximum absolute value, $M$, of $(P Q)$ within $(h, k)$ regardless of spacing, satisfies the inequality

$$
M \leqq \frac{c_{2}^{2}}{a_{1} b_{1}}(2 h)^{m+1}(2 k)^{n+1}
$$

It will be shown that an optimum spacing, $S_{0}$, is that spacing obtained when $x_{u}, y_{v}$ are given by the roots of $T_{m+1}(x / h)=0, T_{n+1}(y / k)=0$, where $T_{k}(z)$ is the polynomial of degree $k$ in $z$, with leading coefficient unity, deviating least from zero within the interval $(-1,1)$ [2]. These roots are:

$$
x_{u}=-h \cos \frac{(2 u+1) \pi}{(2 m+2)} ; \quad y_{v}=-k \cos \frac{(2 v+1) \pi}{2 n+2}
$$


This spacing will also be called Chebyshev spacing. Thus, if $W^{*}=|P|+|Q|$

$$
\left(\frac{c_{1}}{b_{2}} 2^{-m} h^{m+1}+\frac{c_{1}}{a_{2}} 2^{-n} k^{n+1}\right) \leqq W \leqq\left(\frac{c_{2}}{b_{1}} 2^{-m} h^{m+1}+\frac{c_{2}}{a_{1}} 2^{-n} k^{n+1}\right)
$$

where $W$ is equal to $W_{\max }^{*}$ with Chebyshev spacing.

It follows from relations (8) and (10) that

$$
\lim _{h \rightarrow 0} \frac{M}{W}=0, \quad \delta \text { and all } x_{u} / h, y_{v} / k \text { remaining fixed. }
$$

We can now determine a non-zero lower limit on the maximum absolute value of $R_{m n}$ as follows:

We restrict ourselves to such rectangles $(h, k)$ for which $M / W$ is less than unity, which, using relations (8) and (10), is certainly true when

$$
h<\left\{\frac{a_{1} b_{1} c_{1}}{2^{m+n+2} c_{2}^{2}\left(\frac{b_{2} 2^{m}}{\delta^{n+1}}+a_{2} 2^{n}\right)}\right\}^{\frac{1}{1+\mu}} \quad \text { where } \begin{array}{lll}
\mu=m & \text { if } m<n \\
\mu=n & \text { if } n<m .
\end{array}
$$

For any spacing whatsoever, consider equation (5) at the point $\left(x^{*}, y^{*}\right)$ at which $W^{*}$ has its maximum value within $(h, k)$ :

$$
\left|\frac{c_{\xi^{\prime} \eta^{\prime}} R_{m n}}{c_{\xi} c_{\eta}}\right|_{\left(x^{*}, y^{*}\right)}=W_{\max }^{*} \pm \lambda M \quad(0 \leqq \lambda \leqq 1)
$$

Since the extreme values of $|P|$ and $|Q|$ occur independently, it follows that $W_{\max }^{*} \geqq W_{\min }$, where $W_{\min }$ is the lower bound for $W$ in equation (10), i.e., $W_{\min }=\frac{c_{1}}{b_{2}} 2^{-m} h^{m+1}+\frac{c_{1}}{a_{2}} 2^{-n} k^{n+1}$.

Hence,

$$
\left.\left|\frac{c_{\xi^{\prime} \eta^{\prime}}}{c_{\xi} c_{\eta}}\right|\left|R_{m n}\right|\right]_{\left(x^{*}, y^{*}\right)} \geqq W_{\min }-M>0 .
$$

We are thus led to define $R$ by the equation

$$
\frac{c_{2}}{a_{1} b_{1}} R=W_{\min }-M \text {. }
$$

We know then that for any spacing, there exists a point $\left(x^{*}, y^{*}\right)$ within $(h, k)$ for which $\left|R_{m n}\right| \geqq R$. Let $R_{0}=\left|R_{m n}\right|_{\max }$ for Chebyshev spacing.

$$
\lim _{h \rightarrow 0}\left(\frac{R_{0}}{R}\right)=\lim _{h \rightarrow 0}\left(\frac{W+\lambda M}{W_{\min }-M}\right)=\lim _{h \rightarrow 0}\left(\frac{1+\lambda\left(\frac{M}{W}\right)}{1-\left(\frac{M}{W_{\min }}\right)}\right)=1,
$$

$\delta$ and all $x_{u} / h, y_{v} / k$ remaining fixed. Thus, optimum spacing (as defined above) is obtained when $x_{u}, y_{v}$ are respectively the zeros of the Chebyshev polynomials $T_{m+1}(x / h)$ and $T_{n+1}(y / k)=0$.

3. Bi-variate Polynomials $\mathbf{P}_{\mathbf{m n}}(\mathbf{x}, \mathrm{y})$ With Leading Coefficient Unity Deviating Least From Zero Within $(\mathbf{h}, \mathbf{k})$. Let $P_{m n}(x, y)$, defined as in equation (1), but with leading coefficient unity, $\left(a_{m n}=1\right)$, be written as follows: 


$$
P_{m n}(x, y)=x^{m} \phi_{m}(y)+x^{m-1} \phi_{m-1}(y)+\cdots+x \phi_{1}(y)+\phi_{0}(y)
$$

where $\phi_{k}(y)=\sum_{j=0}^{n} a_{k j} y^{j}(k=0,1,2 \cdots m)$ and $\phi_{m}(y)$ has leading term $y^{n}$. Let $y=y_{m}{ }^{*}$ be a value of $y$ for which $\left|\phi_{m}(y)\right|$ reaches its extreme value within $(h, k)$. Then

$$
\left|P_{m n}(x, y)\right|_{\max }>\left|\phi_{m}\left(y_{m}^{*}\right)\right|\left|Q_{m n}\left(x, y_{m n}^{*}\right)\right|_{\max }
$$

where

$$
Q_{m n}\left(x, y_{m}^{*}\right)=x^{m}+\sum_{k=0}^{n-1} \frac{\phi_{k}\left(y_{m}^{*}\right)}{\phi_{m}\left(y_{m}^{*}\right)} \cdot x^{k} .
$$

The least possible values of $\left|\phi_{m}\left(y_{m}^{*}\right)\right|$ and $\left|Q_{m n}\right|_{\max }$ are obtained if

$$
\left\{\begin{array}{l}
\phi_{m}(y)=k^{n} T_{n}(y / k) \\
Q_{m n}\left(x, y_{m}^{*}\right)=h^{m} T_{m}(x / h)
\end{array}\right\} .
$$

For this to be the case it is necessary that

$$
\begin{aligned}
& \frac{\phi_{k}\left(y_{m}^{*}\right)}{\phi_{m}\left(y_{m}^{*}\right)}=\alpha_{k} \\
& k=0,1,2 \cdots(m-1) \\
& m^{*}=1,2, \cdots(n+1)
\end{aligned}
$$

and $\alpha_{k}$ is the coefficient of $x^{k}$ in the Chebyshev polynomial $h^{m} T_{m}(x / h)$. Any of the equations (20) is an algebraic equation in $y_{m}{ }^{*}$ of degree $n$ or less, having at most $n$ real roots. However, $\phi_{m}\left(y_{m}\right)$ in equation (19) has an identical extreme value (except for sign variation) at least at $(n+1)$ points. Hence for equation $(20)$ to hold at these $(n+1)$ points it must represent a set of identities, i.e. $\phi_{k}=\alpha_{k} k^{n} T_{n}(y / k)$, hence

$$
P_{m n}(x, y)=h^{m} k^{n} T_{m}\left(\frac{x}{h}\right) T_{n}\left(\frac{y}{k}\right) .
$$

This polynomial deviates least from zero within $(h, k)$ with maximum deviation, $L$, given by $L=4 h^{m} k^{n} 2^{-(m+n)}$. Note that the lines $x=x_{u}, y=y_{v}$ have replaced the points $x_{u}$ in the one-dimensional theory and that the symmetry $P_{m n}( \pm x, \pm y)=$ $P_{m n}(x, y)=P_{m n}(y, x)$ is preserved. Generalization to $n$-dimensions follows at once.

The above developments arose in the course of work on the synthesis of mechanisms having two degrees of freedom and the author has also utilized with benefit and appreciation the works on interpolation by Biermann [3], Hildebrand [4] and Kunz [5].

Columbia University

New York 27, N. Y.

1. J. F. Stefrensen, Interpolation, The William and Wilkins Co., Baltimore, Md. 1927 (p. 206, eq. 13). Reprint available from Chelsea Publishing Co. Formula goes back to S. Narumi, The Tohoku Math. J. v. 18, 1920, p. 313.

2. N. I. Achirser, translated by Charles J. Hyman, Theory of Approximation, F. Ungar Publishing Co., New York, 1956; in particular p. 55-60 on Chebyshev theory.

3. O. BiermanN, Vorlesungen Ueber Mathematische Naeherungsmethoden, F. Vieweg und Sohn Verlag, Braunschweig, Germany, 1905; in particular p. 138-146 on interpolation involving two variables.

4. F. B. Hildebrand, Introduction to Numerical Analysis, McGraw-Hill Book Co., New York, 1956; in particular p. 279-282, 386-391 on Chebyshev polynomials.

5. K. S. KUNZ, Numerical Analysis, McGraw-Hill Book Co., New York, 1957 ; in particular, Chapter 11, p. 248-274 on interpolation in tables of two or more variables, and equation (11.60) on page 269. 